\title{
Evaluation of T-wave alternans in high-resolution ECG maps recorded during the stress test in patients after myocardial infarction
}

\author{
Dariusz Janusek ${ }^{1}$, Michał Kania ${ }^{1}$, Rajmund Zaczek², Małgorzata Kobylecka ${ }^{3}$, Marek Chojnowski \\ Leszek Królicki³, Grzegorz Opolski², Roman Maniewski ${ }^{1}$
}

\begin{abstract}
${ }^{1}$ Nalecz Institute of Biocybernetics and Biomedical Engineering, Polish Academy of Sciences, Warsaw, Poland

${ }^{2}$ Department of Cardiology, Medical University of Warsaw, Warsaw, Poland

${ }^{3}$ Department of Nuclear Medicine, Medical University of Warsaw, Warsaw, Poland
\end{abstract}

Submitted: 27 September 2013

Accepted: 9 October 2013

Arch Med Sci 2015; 11, 1: 99-105

DOI: 10.5114 /aoms.2014.43893

Copyright (C 2015 Termedia \& Banach

\section{Abstract}

Introduction: Recent studies point to analysis of T-wave alternans as a promising indicator of an increased risk of life-threatening ventricular arrhythmias. In this study the occurrence of T-wave alternans in the high-resolution ECGs recorded during the exercise stress test and scintigraphic tests (SPECT) in patients with ischemic heart disease was examined.

Material and methods: The study group consisted of 33 patients after myocardial infarction. In the group of patients after myocardial infarction and with low left ventricular ejection fraction correlations of $70 \%$ between the test results of T-wave alternans and SPECT and $60 \%$ between the test results of T-wave alternans and stress test were found.

Results: In the group of patients after myocardial infarction but with high left ventricular ejection fraction correlations were respectively $39 \%$ and $48 \%$. The analysis of the electrocardiographic maps showed a strong dependence of this correlation on the T-wave alternans amplitude and location of the ECG measuring electrode on the chest. The results might suggest that in patients after myocardial infarction and at increased risk for sudden cardiac death T-wave alternans may also provide information about cardiac electrical instability associated with ischemia.

Conclusions: It can also be assumed that the position of the electrode where the highest level of the T-wave alternans was detected can indicate the location of the ischemic region of the heart.

Key words: T-wave alternans, stress test, single-photon emission computed tomography, arrhythmia, sudden cardiac death.

\section{Introduction}

The development of reliable indicators of the sudden cardiac death $(S C D)$ risk in patients with ischemic heart disease is one of the most important challenges for modern cardiology. Research into cardiac electrical signals, the analysis of which can detect abnormal heart function, is of particular interest. Currently, there is no generally accepted non-invasive indicator of SCD risk other than the left ventricular ejection fraction [1, 2]. The most effective indicators include the occurrence of arrhythmic

\author{
Corresponding author: \\ Dariusz Janusek PhD \\ Nalecz Institute \\ of Biocybernetics and \\ Biomedical Engineering \\ Polish Academy of Sciences \\ $4 \mathrm{Ks}$. Trojdena St \\ 02-109 Warsaw, Poland \\ Phone: +48 226599143 ext 104 \\ Fax: +48 226597030 \\ E-mail: djanusek@ibib.waw.pl
}




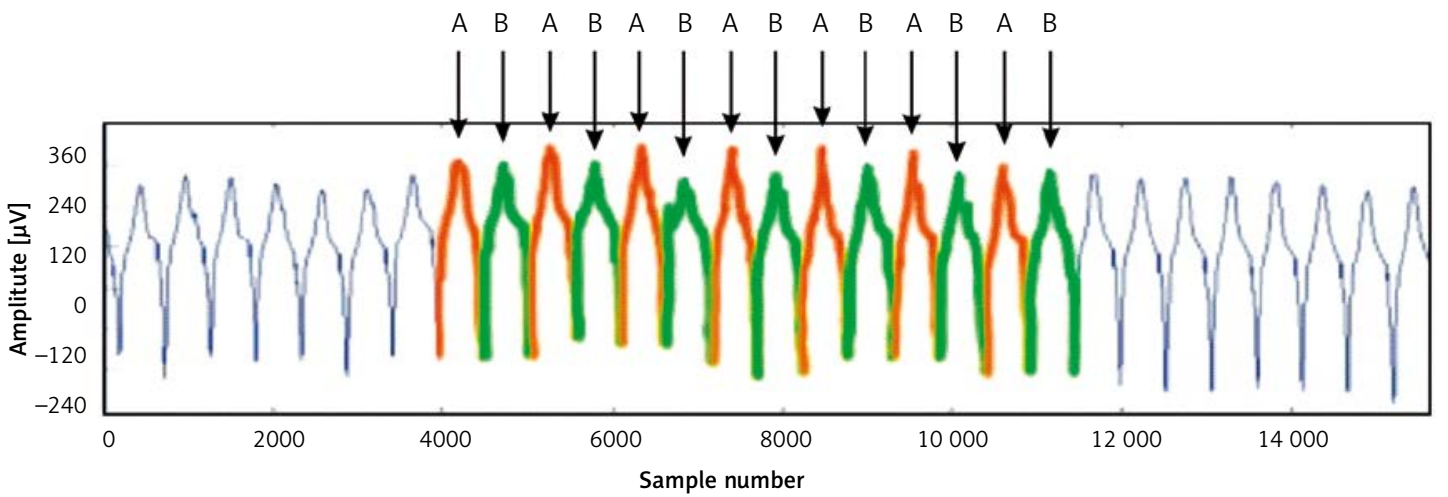

Figure 1. Fragment of ECG signal with present T-wave alternans phenomenon

events in Holter ECG recordings, the occurrence of ventricular late potentials in the averaged ECG, heart rate variability, the duration and dispersion of ventricular repolarization, and the left ventricular ejection fraction.

Recent studies point to analysis of T-wave alternans (TWA) as a promising indicator of an increased risk of life-threatening ventricular arrhythmias [3-5]. T-wave alternans is an electrocardiographic abnormality involving a disorder of ventricular repolarization in the consecutive regular heart beats. It electrically describes cyclic changes, occurring in every second heart beat, in the shape and amplitude of the T-wave. Figure 1 shows an example of an ECG waveform with TWA. Alternately appearing T-waves of a higher $(A)$ and lower (B) amplitude are visible.

One of the first computer-assisted attempts to assess the repolarization process by examination of T-wave alternans was the spectral method proposed by Adam et al. [6] and then modified by Smith et al. [2]. The TWA was assessed on the basis of the averaged power spectra of signals consisting of time-synchronized samples of consecutive T-waves, calculated for 128 heart beats [2]. T-wave alternans is an indicator of the risk of SCD which allows one to detect lesions well in advance and initiate treatment and the healing process in the early stages of the disease. It is assumed that it is the expression of dynamic heterogeneity of cardiac repolarization. The mechanisms of the formation as well as the course of the repolarization alternation process and its links with the rest of the phenomena that occur in the myocardium are not fully known and are still under investigation. Based on the results of the previous works, it is known that the process can be to varying degrees nonstationary. The prognostic value of TWA has been proven in patients with post-MI cardiac failure [7-9], chronic heart failure [10] and dilated cardiomyopathy [11].

Treadmill or bicycle exercise testing, using 12lead ECG monitoring, remains a useful option in patients with suspected stable coronary artery disease because of its simplicity and widespread availability [12]. An increased effort on a treadmill or cycle ergometer is intended to produce controlled myocardial ischemia due to oxygen deficiency. No increase in pulse rate or systolic blood pressure adequate to the degree of physical activity indicates advanced coronary artery disease associated with impaired left ventricular function. Exercise-induced ischemia may be local. The application of electrocardiographic imaging in the form of maps of potentials on the surface of the human body allows one to estimate the location of these changes [13, 14].

Greater sensitivity in the diagnosis of coronary heart disease is obtained by applying perfusion scintigraphy (SPECT) [15]. An analysis of scintigraphic images recorded during the resting phase and during exercise allows for a local assessment of myocardial blood perfusion, enabling one to determine the location and extent of ischemia. At the same time, perfusion scintigraphy is used to assess the presence of reversible myocardial ischemia in patients after myocardial infarction. The size of the area covered by necrosis or reversible ischemia determines the prognosis of a patient and any decision regarding coronary revascularization.

The aim of this study was to evaluate the T-wave alternans in the high-resolution ECG maps recorded during the stress test as a risk marker of cardiac ischemia in MI patients. The SPECT was used as a reference method.

\section{Material and methods}

\section{Examined patients}

The study group consisted of 33 patients after myocardial infarction. The characteristics of the group are shown in Table I.

Patients eligible for this study had current indications for the diagnosis of coronary artery disease using the cardiac SPECT examination. The TWA recordings were made during the stress test on a cycle ergometer, which is part of the SPECT 
Table I. The study group of patients after myocardial infarction

\begin{tabular}{|c|c|c|c|c|c|c|c|}
\hline & $\begin{array}{l}\text { Number of } \\
\text { patients }\end{array}$ & Age & $\begin{array}{l}\text { Left ventricular } \\
\text { ejection frac- } \\
\text { tion (LVEF) }\end{array}$ & LVDD & BMI & STEMI & NSTEMI \\
\hline LVEF $\leq 40$ & 10 & 69 (SD 8.59) & 36.70 (SD 3.83) & $5.13($ SD 0.81) & 26 (SD 3.65) & 8 (80\%) & $2(20 \%)$ \\
\hline \multirow[t]{2}{*}{ LVEF > 40} & 23 & 58 (SD 8.45) & 52.78 (SD 7.05) & 5.00 (SD 0.57) & 28 (SD 4.13) & 19 (83\%) & $4(17 \%)$ \\
\hline & & $p<0.005$ & $p<0.005$ & NS & NS & & \\
\hline
\end{tabular}

protocol. All the patients gave their consent to be included in the study. The study was carried out at the Central Clinical Hospital of the Medical University of Warsaw. The examinations were approved by the University Bioethics Committee.

\section{Signal measurement and analysis}

The ECG signals were recorded using Active-Two, a multi-lead ECG system (Biosemi, Amsterdam, Netherlands). Sixty-four active electrodes were positioned according to the University of Amsterdam system [16]. The applied electrode arrangement is shown in Figure 2.

The ECG signals were acquired during the stress test carried out on the cycle ergometer in an unshielded room in the hospital. The stress test protocol started with the initial load of $50 \mathrm{~W}$, which was then increased by $25 \mathrm{~W}$ every $2 \mathrm{~min}$. For the study of T-wave alternans this protocol was modified. At the moment of obtaining a heart rate of 100 beats per minute the standard stress test protocol was interrupted for $2 \mathrm{~min}$. At that time, the load was manually adjusted in such a way as to obtain a constant heart rate of 100 beats per min ( \pm 5 beats per minute). For the assessment of the T-wave alternans testing results, a part of the electrocardiogram recorded during manual control was subjected to further analysis. The fluctuations of the isoelectric line were eliminated from the ECG using the third-degree polynomial method [17]. The QRS complex was detected by the algorithm developed by Jiapu Pan and Willis Tompkins [18]. The location of the T-wave was determined based on an array of data describing the relationship between the QT and RR times [19]. All the disrupted

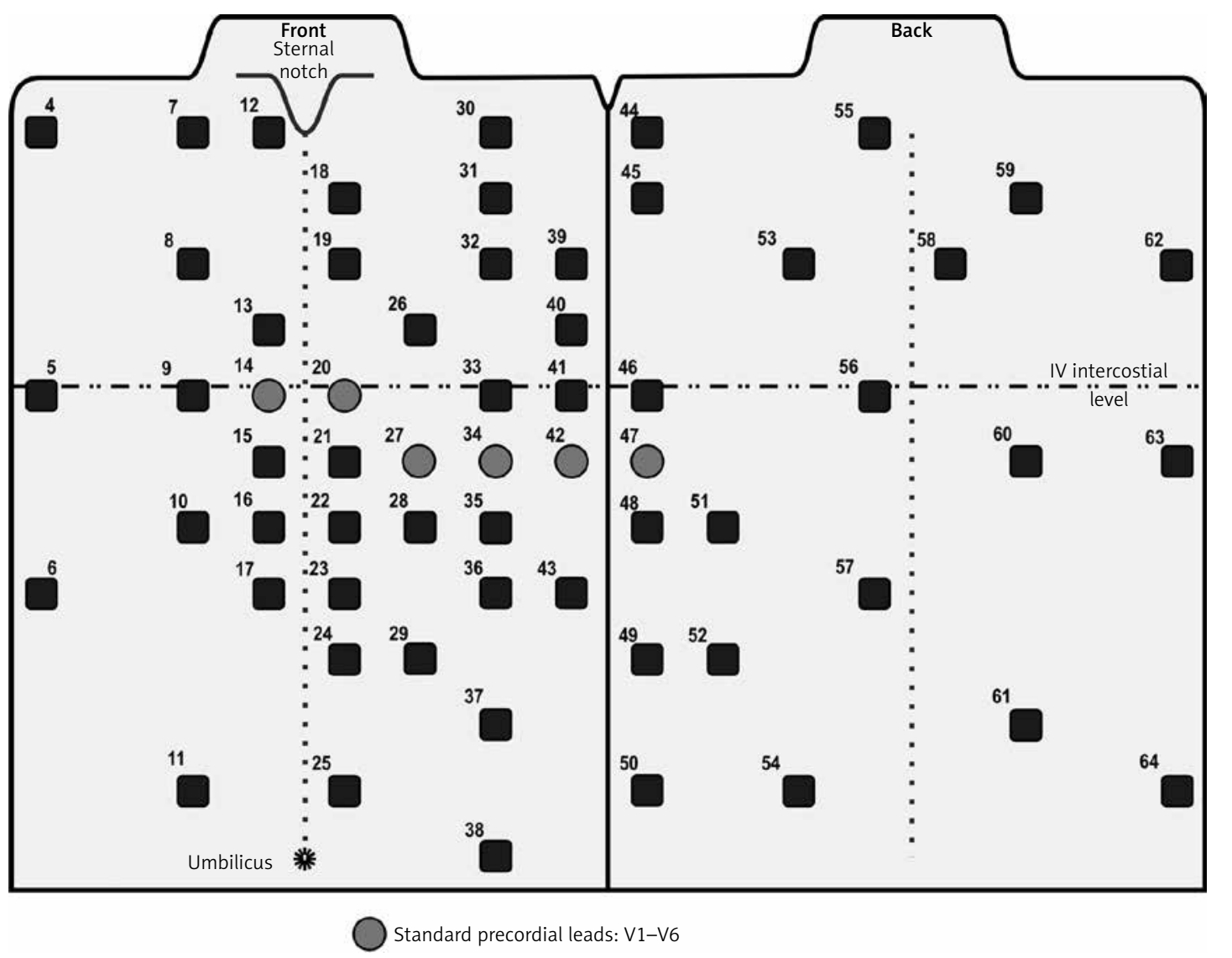

Figure 2. Arrangement of ECG electrodes 
cycles were eliminated from the recorded ECG signal. The alternans ratio was determined using the spectral method with incoherent averaging. The power spectrum was determined separately for the data vectors of the consecutive time synchronized T-wave samples (a single vector describes changes in the amplitude of a determined T-wave sample in the consecutive heart beats). The cumulative power spectrum was obtained by averaging the squares of the FFT modules calculated for each vector (the spectra of vectors containing signals of alternans for the consecutive samples of the analyzed T-waves were averaged). The amplitude of T-wave alternans in the cumulative power spectrum was represented by a spectrum band with the frequency of 0.5 cycles/beat. The T-wave alternans coefficient $(A R)$ was determined according to the formula:

$$
A R=\frac{P_{0.5}-\overline{\text { noise }}}{\sigma_{\text {noise }}} ;
$$

where: $P_{0.5}$ is the power of the signal at the TWA frequency of 0.5 cycles/beat; noise, $\sigma_{\text {noise }}$ respectively, are the mean and the standard deviation of the power of noise assessed in the window of the cumulative power spectrum located outside the alternans frequency band (from 0.35 to 0.45 cycles/beat).

The value of the T-wave alternans coefficient equal to or greater than 2.5 [a.u.] indicates the detection of a diagnostically relevant T-wave alternans [7]. The values of the T-wave alternans coefficients were determined independently in all the 64 leads.

The exercise stress test was continued until a diagnostic result was obtained [18]. The result was classified as non-diagnostic when it was necessary to interrupt the test. The stress test was classified as positive in the case of:

- lowering of the ST segment by $0.1 \mathrm{mV}$ (60 ms after the J point) or more in two adjacent leads, - elevation of the ST segment by $0.2 \mathrm{mV}$,

- the occurrence of chest pain,

- the occurrence of ischemia symptoms,

- the necessity of test interruption at a low load,

- instances of life-threatening cardiac arrhythmias.

All patients underwent myocardial perfusion scintigraphy to have the myocardial blood supply assessed at rest and during stress testing. The SPECT data were acquired 60 min after intravenous administration of $500-740 \mathrm{MBq}$ technetium-99m MIBI. The radiopharmaceutical, with myocardial distribution proportional to the blood supply, was injected during the stress test at the time of maximum effort. The images were acquired with the aid of a two-head gamma camera (Varicam Elscint) for $20 \mathrm{~s}$ in 32 steps between the right anterior $45^{\circ}$ and left posterior $45^{\circ}$ (zoom 1.28) then stored in a $64 \times 64$ matrix. Transverse slices of acquired data were reconstructed by filtered back projection using a Butterworth filter order 5 and cut-off frequency of 0.35 Nyquist. Images were re-orientated according to the long axis of the left ventricle. The test was considered positive if the myocardial reversible perfusion defect was greater than $10 \%$ of the muscle of the left ventricle.

\section{Results}

The distribution of the diagnostically relevant T-wave alternans coefficients obtained from the electrocardiographic maps for selected patients is shown in Figure 3. Only coefficients with values above the threshold of diagnostically relevant T-wave alternans (2.5 [a.u.]) are presented.

There were maps for patients in whom the value of the T-wave alternans coefficient exceeded the aforementioned diagnostic threshold in at least one electrocardiographic lead. The analysis of the T-wave alternans testing results showed that a diagnostically relevant value of the alternans coefficient most frequently occurred in leads 30 and 60. The study group was divided into two subgroups: TWA (+) - the patients in whom the value of the T-wave alternans coefficient exceeded 2.5 [a.u.] in at least one lead; and TWA (-) - the patients in whom the value of the T-wave alternans coefficient was below the medical diagnostic threshold. There was a maximum value of the T-wave alternans coefficient calculated in one of the 64 leads, determined independently for each patient.

In all analyzed signals from the patients from the study group three tests were performed: T-wave alternans analysis, stress test and SPECT examination. The results were classified as positive (in the case of TWA medically significant T-wave amplitude alternations were detected; in the case of stress test and SPECT myocardial ischemia was detected) or negative (abnormalities were not detected). The results were marked respectively 1 and 0 and comparison of the results was performed. Detection compatibility was marked as positive when two results were positive (1 s). The data about positive detections and detection compatibility are shown in Table II.

\section{Discussion}

The development of high-resolution measurement techniques and computer-assisted advanced analysis of the ECG data allowed for accurate assessment of abnormalities in different parts of the ECG and their relations to heart disease. Unfortunately, ventricular repolarization abnormalities, reflecting pathological T-wave changes, are still poorly understood. The most common method of ventricular repolarization analysis is the measure- 

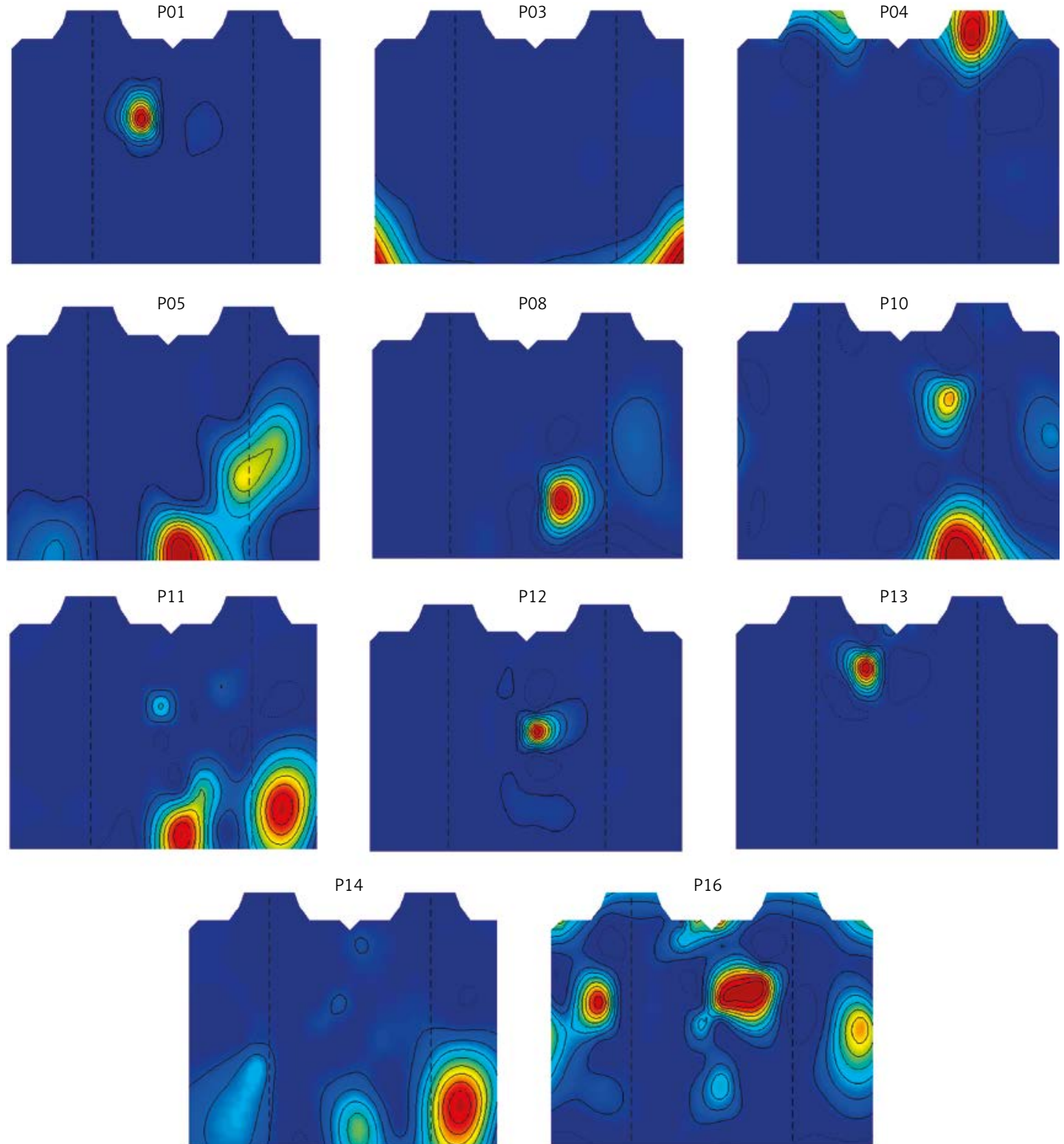

P16

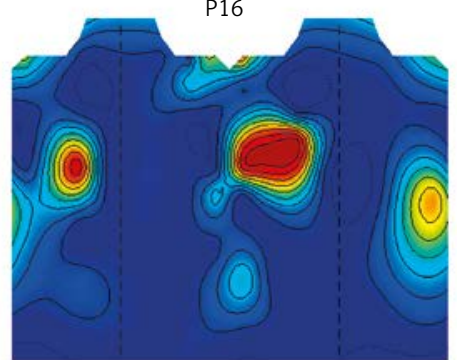

Figure 3. Maps of alternans coefficient values on the thorax of the selected patients with diagnostically relevant T-wave alternans

ment of the QT interval. This test does not give any information about its instantaneous abnormalities, which may occur without significant prolongation of the QT interval, though it determines the total duration of ventricular repolarization. The major problem in this measurement is to ensure the accuracy and repeatability of determining the T-wave end and its relation to HR.

The SPECT test was used in our study for the evaluation of ischemia in patients after myocardial infarction. The stress test was performed during the stress part of SPECT. At that time, the T-wave alternans test was being carried out as well. It was necessary because TWA is a phenomenon strongly dependent on heart rate. To diagnostically evaluate the T-wave alternans test results, it is neces- sary to carry out ECG recordings at the heart rate of 100 beats per minute.

The analysis of the maps of T-wave alternans coefficient values showed strong differentiation in the level of the received TWA signals on the location of the receiving electrode on the thorax. In some of the examined patients the T-wave alternans would not have been detected using the traditional standard twelve-lead system, as a diagnostically important signal level was recorded in the leads outside the standard layout [20-22].

It should be stressed that multiple lead electrocardiography, like the commonly used standard ECG, is non-invasive, safe and inexpensive for the patient. It can be assumed that the location of the infarction scar on the heart has a strong influence 
Table II. Results of T-wave alternans test, ECG stress test and SPECT and detection compatibility

\begin{tabular}{|c|c|c|c|c|c|c|}
\hline & \multicolumn{3}{|c|}{ Positive test result } & \multicolumn{3}{|c|}{ Detection compatibility } \\
\hline & $\begin{array}{c}\text { T-wave alternans } \\
\text { (TWA) }\end{array}$ & $\begin{array}{l}\text { Stress test } \\
\text { (ST) }\end{array}$ & SPECT & TWA vs. SPECT & TWA vs. ST & ST vs. SPECT \\
\hline LVEF $\leq \mathbf{4 0}$ & $7(70 \%)$ & $5(50 \%)$ & $6(60 \%)$ & $7(70 \%)$ & $6(60 \%)$ & $5(50 \%)$ \\
\hline LVEF > 40 & $8(35 \%)$ & $12(52 \%)$ & $12(52 \%)$ & $11(48 \%)$ & 9 (39\%) & $13(57 \%)$ \\
\hline
\end{tabular}

on the strength of the received T-wave alternans signal for specific locations of the electrodes.

The comparison of the results of the T-wave alternans test with the stress test result and then with the SPECT test result showed a significant correlation dependence (respectively $60 \%$ and $70 \%$ concordant) in the patients after myocardial infarction and with low left ventricular ejection fraction, in contrast to the study performed in the patients with a high left ventricular ejection fraction (respectively $39 \%$ and $48 \%$ concordant). This may indicate higher detection sensitivity of the TWA and SPECT methods in detecting ischemia, which is associated with an increased risk of life-threatening ventricular arrhythmias.

The area of ischemia, especially the post-infarct scar, may influence the propagation pattern of the depolarization and the repolarization waves in the heart muscle. The resulting disturbances may create condition blocks and form the re-entry-type arrhythmia which may cause cardiac death. The causes of the T-wave alternans in electrocardiograms has not yet been clearly defined. It can be assumed that the changes in propagation caused by ischemia may create conditions conducive to the formation of TWA. It is necessary to carry out long-term observation of the patients included in the study group and on this basis separate the patients at increased risk of ventricular arrhythmia (a classification on the basis of previous arrhythmic events or mortality in long-term observation).

In conclusion, the results of the tests carried out in the patients with ischemic heart disease after myocardial infarction showed a significant correlation of the T-wave alternans and results of the ECG stress test and the SPECT test in the patients with low left ventricular ejection fraction, unlike the results obtained in the patients with high ventricular ejection fraction. The analysis of the electrocardiographic maps showed a strong dependence of this correlation on the T-wave alternans amplitude and location of the ECG measuring electrode on the chest.

The results might suggest that in patients after myocardial infarction and at increased risk for sudden cardiac death, T-wave alternans may also provide information about cardiac electrical instability associated with ischemia. It can also be assumed that the position of the electrode where the highest level of the T-wave alternans was detected can indicate the location of the ischemic region of the heart.

\section{Acknowledgments}

The study was funded by the National Science Centre within the research projects NN518 504399 and DEC-2011/01/B/ST7/06801.

\section{Conflict of interest}

The authors declare no conflict of interest.

\section{References}

1. Fereniec M, Stix G, Kania M, Mroczka T, Janusek D, Maniewski R. Risk assessment of ventricular arrhythmia using new parameters based on high resolution body surface potential mapping. Med Sci Monitor 2011; 17: MT26-33.

2. Smith JM, Clancy EA, Valeri CR, Ruskin JN, Cohen RJ. Electrical alternans and cardiac electrical instability. Circulation 1988; 77: 110-21.

3. Verrier RL, Nearing BD, Ghanem RN, et al. Elevated T-wave alternans predicts nonsustained ventricular tachycardia in association with percutaneous coronary intervention in ST-segment elevation myocardial infarction (STEMI) patients. J Cardiovasc Electrophysiol 2013; 24: 658-63.

4. Zipes DP, Camm AJ, Borggrefe M, et al. ACC/AHA/ESC 2006 guidelines for management of patients with ventricular arrhythmias and the prevention of sudden cardiac death: a report of the American College of Cardiology/American Heart Association Task Force and the European Society of Cardiology Committee for Practice Guidelines (Writing Committee to Develop Guidelines for Management of Patients With Ventricular Arrhythmias and the Prevention of Sudden Cardiac Death). J Am Coll Cardiol 2006; 48: e247-346.

5. Janusek D, Pawlowski Z, Maniewski R. Evaluation of the T-wave alternans detection methods: a simulation study. Anatol J Cardiol 2007; 7 Suppl 1: 116-9.

6. Adam DR, Smith JM, Akselrod S, Nyberg S, Powell AO, Cohen RJ. Fluctuations in T-wave morphology and susceptibility to ventricular fibrillation. J Electrocardiol 1984; 17: 209-18.

7. Rosenbaum DS, Jackson LE, Smith JM, Garan H, Ruskin JN, Cohen RJ. Electrical alternans and vulnerability to ventricular arrhythmias. N Engl I Med 1994; 330: 235-41.

8. Zareba W, Moss AJ. Noninvasive risk stratification in postinfarction patients with severe left ventricular dysfunction and methodology of the MADIT II noninvasive electrocardiology substudy. J Electrocardiol 2003; 36: 101-8. 
9. Hohnloser S, Ikeda T, Bloomfield D, Dabbous O, Cohen R. T-wave alternans negative coronary patients with low ejection and benefit from defibrillator implantation. Lancet 2003; 362: 125-6.

10. Klingenheben T, Zabel M, D'Agostino R, Cohen R, Hohnloser S. Predictive value of T-wave alternans for arrhythmic events in patients with congestive heart failure. Lancet 2000; 356: 651-2.

11. Hohnloser SH, Klingenheben T, Bloomfield D, Dabbous O, Cohen RJ. Usefulness of microvolt T-wave alternans for prediction of ventricular tachyarrhythmic events in patients with dilated cardiomyopathy: results from a prospective observational study. J Am Coll Cardiol 2003; 41: 2220-4.

12. Fox K, Garcia MAA, Ardissino D, et al. Guidelines on the management of stable angina pectoris: executive summary The Task Force on the Management of Stable Angina Pectoris of the European Society of Cardiology. Eur Heart J 2006; 27: 1341-81.

13. Mirvis DM. Current status of body surface electrocardiographic mapping. Circulation 1987; 75: 684-8.

14. Janusek D, Kepski R, Kania M, Fereniec M, Maniewski R (eds.). Spatial distribution of T-wave alternans (Abstract). Computers in Cardiology 2007; Durham, North Carolina, USA. IBIB.

15. Elhendy A, Bax JJ, Poldermans D. Dobutamine stress myocardial perfusion imaging in coronary artery disease. J Nucl Med 2002; 43: 1634-46.

16. Sippens Groenewegen A, Spekhorst H, Van Hemel N, et al. Localization of the site of origin of postinfarction ventricular tachycardia by endocardial pace mapping. Body surface mapping compared with the 12-lead electrocardiogram. Circulation 1993; 88: 2290-306.

17. Meyer C, Keiser H. Electrocardiogram baseline noise estimation and removal using cubic splines and statespace computation techniques. Comp Biomed Res 1977; 10: 459-70

18. Pan J, Tompkins WJ. A real-time QRS detection algorithm. Biomedical Engineering, IEEE Transactions on 1985; 32: 230-6.

19. Bazett H. An analysis of the time relations of electrocardiograms. Ann Noninv Electrocardiol 2006; 2: 177-94.

20. Janusek D, Kania M, Zaczek R, et al. Application of wavelet based denoising for T-wave alternans analysis in high resolution ECG maps. Measurement Science Review 2011; 11: 181-4.

21. Selvaraj RJ, Suszko AM, Subramanian A, et al. Body surface projection of action potential duration alternans: a combined clinical-modeling study with implications for improving T-wave alternans detection. Heart Rhythm 2009; 6: 1211-9.

22. Kania M, Zaczek R, Zavala-Fernandez $H$, et al. ST-segment changes in high-resolution BSPM measured during exercise to assess myocardial ischemia: a pilot study. Arch Med Sci 2014; 10: 1086-90. 